\title{
Functional Polymorphisms in hOGGl Gene and Neuroblastoma Risk in Chinese Children
}

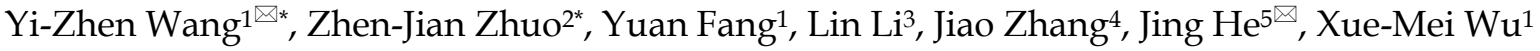 \\ 1. Department of Pathology, Anhui Provincial Children's Hospital, Hefei 230051, Anhui, China \\ 2. School of Chinese Medicine, Faculty of Medicine, The Chinese University of Hong Kong, Hong Kong 999077, China \\ 3. Clinical Laboratory, Anhui Provincial Children's Hospital, Hefei 230051, Anhui, China \\ 4. Department of Pediatric Surgery, the First Affiliated Hospital of Zhengzhou University, Zhengzhou 450052, Henan, China \\ 5. Department of Pediatric Surgery, Guangzhou Institute of Pediatrics, Guangzhou Women and Children's Medical Center, Guangzhou Medical University, \\ Guangzhou 510623, Guangdong, China \\ "These authors contributed equally to this work.
}

$\triangle$ Corresponding authors: Yi-Zhen Wang, Department of Pathology, Anhui Provincial Children's Hospital, 39 East Wangjiang Road, Hefei 230051, Anhui, China, Email: ywff018@163.com; or Jing He, Department of Pediatric Surgery, Guangzhou Institute of Pediatrics, Guangzhou Women and Children's Medical Center, Guangzhou Medical University, 9 Jinsui Road, Guangzhou 510623, Guangdong, China, Email: hejing198374@gmail.com.

(C) Ivyspring International Publisher. This is an open access article distributed under the terms of the Creative Commons Attribution (CC BY-NC) license (https://creativecommons.org/licenses/by-nc/4.0/). See http://ivyspring.com/terms for full terms and conditions.

Received: 2018.06.19; Accepted: 2018.09.09; Published: 2018.10.31

\begin{abstract}
Neuroblastoma is a lethal tumor of the sympathetic nervous system. 8-Hydroxydeoxyguanine (8-OH-dG) formation is a common seen type of oxidative DNA damage, which could be repaired by human oxoguanine glycosylase 1 (hOGGl). To explore the contributing role of hOGGl gene single nucleotide polymorphisms (SNPs) in neuroblastoma risk, we performed a case-control study by genotyping three SNPs (rs1052133 G>C, rs159153 T>C, rs293795 A>G) in hOGGl gene. A total of 512 neuroblastoma cases and 1076 cancer-free controls were enrolled from three medical centers in China. The hOGGl gene polymorphisms were determined using TaqMan real-time PCR. The results showed that only the rs $1052133 \mathrm{G}>\mathrm{C}$ polymorphism was associated with neuroblastoma risk [GC vs. GG: adjusted odds ratio $(O R)=0.64,95 \%$ confidence interval $(C l)=0.51-0.81, P=0.0002$; dominant model: adjusted $\mathrm{OR}=0.71,95 \% \mathrm{Cl}=0.57-0.88, P=0.002]$. Moreover, subjects carrying 1,2 , or 1-3 protective genotypes have less opportunity to develop neuroblastoma, in comparison to those without protective genotypes. Stratified analysis revealed that $\mathrm{rs} 1052133 \mathrm{GC} / \mathrm{CC}$ carriers were less likely to develop neuroblastoma in subgroups of age $>18$ months, males, tumor that develops from retroperitoneal, mediastinum and clinical stage $1+\|+4$ s. Our results indicate that hOGGl rs1052133 G>C polymorphism is associated with decreased risk of neuroblastoma. However, the exact biological mechanism awaits further research.
\end{abstract}

Key words: neuroblastoma, hOGG1, polymorphism, susceptibility, DNA repair

\section{Introduction}

Neuroblastoma, a malignancy mainly diagnosed before age 5 , is the most common extracranial solid tumor in infants [1]. It is a cancer that mainly develops from the adrenal medulla and the sympathetic ganglia [2]. Neuroblastoma presents approximately $7 \%$ of all pediatric malignancies and $15 \%$ of all childhood cancer deaths [2, 3]. Neuroblastoma is remarkable for its widely variable clinical heterogeneity [1]. Intermediate- or low- risk neuroblastoma is highly curable, which might spontaneous regress without chemotherapy. However, high-risk neuroblastoma succumbs to therapy-resistant disease [4]. The 5-year survival rate of high-risk neuroblastoma, which accounts for nearly $50 \%$ of all cases, still less than $40 \%$ despite intensive, multi-modal therapy [4-7].

The factors that impact the neuroblastoma risk are only partially defined. Several environmental factors have been implicated in the initiation of neuroblastoma, yet still lack strict associations [8,9]. 
Growing evidence indicated that neuroblastoma results from the combination of genetic factors and environmental factors. The etiology of familiar neuroblastoma is mainly attributed to the mutations of $P H O X 2 B$ [10] and ALK [11, 12] genes. Genome-wide association studies (GWASs) have revealed that risk alleles within genes TP53, HACE1, BARD1, LIN28B, LMO1, and CASC15 are associated with sporadic neuroblastoma risk [13-17]. As neuroblastoma is a heterogeneous and complex disease, identification of more genetic variants in influencing neuroblastoma risk could help to better managing this disease.

Human genome is continuously exposed to the assaults by different exogenous and endogenous carcinogens or mutagens [18]. Sustained oxidative stress, such as exposure to smoke, induces oxidative DNA adducts [19]. 8-hydroxy-2-deoxyguanine (8-OH-dG) is a major form of oxidative DNA damage with highly mutagenic character [20]. It would cause GC to TA transversions, if not excised on DNA replication [21]. An increase in 8-OH-dG content in DNA is associated with tumor initiation and progression [22]. Thus, it is important to preserve genome integrity through the repair of damaged DNA. Human 8-oxoguanine glycosylase 1 (hOGG1) is a multifunctional DNA glycosylase that participates in the repair of DNA oxidative damage [23]. This enzyme could specifically recognize the $8-\mathrm{OH}-\mathrm{dG}$ damage and then efficiently catalyze and remove the damage [24].

hOGG1 gene is located to chromosome 3p25 with eight exons. Several single nucleotide polymorphisms (SNPs) of the hOGG1 gene have been identified, and their contributing roles in cancer risk have been evaluated in many studies [25-27]. However, few studies have been conducted to evaluate the association of hOGG1 gene polymorphisms and neuroblastoma risk. To determine whether common genetic variants of hOGG1 gene confer risk for neuroblastoma, we performed a case-control study in a Chinese Han population from three regions.

\section{Materials and Methods}

\section{Study populations}

A total of 512 neuroblastoma cases and 1076 healthy controls from three centers (Guangzhou Women and Children's Medical Center, The First Affiliated Hospital of Zhengzhou University, and Anhui Provincial Children's Hospital) were included in this study. To be specific, 275 cases and 531 controls were enrolled from Guangzhou, Guangdong; 118 cases and 281 controls were recruited from Zhengzhou, Henan [28-30]; and 119 cases and 264 controls were enrolled from Hefei, Anhui. We obtained signed informed consent before the study from every participant or his/her parents. The study protocol was approved by the Institutional Review Board of the above three hospitals. More details of the enrollment procedure were provided in our previous publication [31-33].

\section{SNP selection and genotyping}

The included potentially functional candidate SNPs were selected as follows: located in the $5^{\prime}$ untranslated region, 3' untranslated region, 5' flanking region, and exon of hOGG1 gene. NCBI dbSNP database (http://www.ncbi.nlm.nih.gov/ projects/SNP) and SNPinfo (http://snpinfo.niehs. nih.gov/snpfunc.htm) online software were used to perform the above selection. We chose three potentially functional SNPs in the hOGG1 gene (rs1052133 G>C, rs159153 T>C, and rs293795 A>G) for analysis. The rs1052133 G>C and rs293795 A>G are located within miRNA binding sites, and the rs159153 $\mathrm{T}>\mathrm{C}$ is located in transcription factor binding sites (TFBS). Genomic DNA was derived from EDTAperipheral blood by using TIANamp Blood DNA Kit (TianGen Biotech Co. Ltd., Beijing, China). The genotyping of all the subjects was carried out using TaqMan real-time PCR (Applied Biosystems), according to the manufacturer's protocols [34-36]. In each plate, eight negative controls with water were used for quality control. Investigators were blinded to the status of all case and control samples. $10 \%$ of samples were randomly selected for a second genotyping, and the genotype concordance rate was $100 \%$.

\section{Statistical analysis}

Departures from Hardy-Weinberg equilibrium were assessed for each SNP in controls by goodnessof-fit $\chi^{2}$ test. Two-sided chi-square test and $t$ test were conducted, as appropriate to compare the demographic variables and allele frequencies between the two groups. The odds ratio (OR), and the corresponding 95\% confidence interval (CI) for each SNP were calculated. Logistic regression analysis was performed to determine the correlation between SNPs and neuroblastoma risk. Statistical adjustment for age and gender was performed. The version 9.4 SAS software (SAS Institute, Cary, NC) was used to perform analyses. The significant threshold was $P<0.05$.

\section{Results}

\section{Population characteristics}

A detailed description of the study sample from Guangzhou and Zhengzhou was presented previously [33, 37, 38]. The population demographics of the cases and controls from Hefei, Anhui province were presented in Table 1. No significant differences 
were observed in terms of age $(P=0.507)$ and gender $(P=0.941)$ between the case and the control groups. Of them, 45, 52, 14, and 8 patients were classified as clinical stages I, II, III, and IV neuroblastoma, respectively. Among these cases, 43 lesions occurred in adrenal gland, 41 in retroperitoneal region, 26 in mediastinum, and 9 in other region.

\section{Correlation of hOGGI gene polymorphisms with neuroblastoma susceptibility}

The genotype frequencies of hOGG1 associated with neuroblastoma risk were shown in Table 2. Overall, we found an inverse association between rs1052133 C allele and neuroblastoma risk (GC vs. GG: adjusted OR=0.64, 95\% CI=0.51-0.81, $P=0.0002$; dominant model: adjusted OR=0.71, 95\% $\mathrm{CI}=0.57-0.88, \quad P=0.002)$. No statistically significant associations were detected regarding the rs159153 $\mathrm{T}>\mathrm{C}$, rs293795 $\mathrm{A}>\mathrm{G}$ polymorphisms and neuroblastoma risk. We also presented the combined effects of protective genotypes on neuroblastoma risk. We found that individuals carrying 1 or 2 protective genotypes have a significant decreased neuroblastoma risk in comparison to those without protective genotypes with adjusted $\mathrm{OR}=0.78,95 \% \mathrm{CI}=0.61-0.99$, $P=0.039$; or adjusted $\mathrm{OR}=0.69$; 95\% $\mathrm{CI}=0.50-0.95$, $P=0.024$, respectively. Similarly, individuals with 1-3 combined protective genotypes of hOGG1 were also less likely to develop neuroblastoma (adjusted $\mathrm{OR}=0.75 ; 95 \% \mathrm{CI}=0.60-0.94, P=0.012)$, compared with those with 0 protective genotypes.

\section{Stratification analysis}

Table 3 showed results from stratification analyses of association between hOGG1 genotypes and neuroblastoma risk, stratified by age, gender, tumor sites of origin and clinical stages. For age, hOGG1 rs1052133 GC/CC genotype was significantly associated with decreased neuroblastoma risk among those $>18$ months (GC/CC vs. GG: adjusted $\mathrm{OR}=0.66$; $95 \% \mathrm{CI}=0.50-0.87)$. Significant inverse associations were also detected in subgroups of males (GC/CC vs. GG: adjusted $\mathrm{OR}=0.63 ; 95 \% \mathrm{CI}=0.47-0.85)$, tumor that develops from retroperitoneal (GC/CC vs. GG: adjusted $\mathrm{OR}=0.58 ; 95 \% \mathrm{CI}=0.40-0.85)$, tumor that develops from mediastinum (GC/CC vs. GG: adjusted $\mathrm{OR}=0.66 ; 95 \% \mathrm{CI}=0.46-0.96)$ and clinical stage I+II+4s (GC/CC vs. GG: adjusted OR=0.64; $95 \%$ $\mathrm{CI}=0.48-0.85)$. In the stratified analysis of cumulative effects of protective genotypes, we found that the presence of 1-3 protective genotypes were protected from neuroblastoma in subgroups of age $>18$ months (adjusted $\mathrm{OR}=0.71 ; \quad 95 \% \quad \mathrm{CI}=0.53-0.93$ ), males (adjusted $\mathrm{OR}=0.71 ; 95 \% \mathrm{CI}=0.53-0.96$ ), tumor that develops from retroperitoneal (adjusted $\mathrm{OR}=0.63$; 95\% CI=0.43-0.92), and clinical stage I+II+4s (adjusted $\mathrm{OR}=0.71 ; 95 \% \mathrm{CI}=0.54-0.95$ ).

\section{Discussion}

To the best of our knowledge, this study was the largest-scale case-control study to date to investigate the impact of hOGG1 gene SNPs on the neuroblastoma risk in Chinese population. Our data demonstrated that a functional polymorphism rs1052133 G>C in hOGG1 gene presented significant associations with decreased neuroblastoma risk.

Table 1. Demographic characteristics for neuroblastoma patients and cancer-free controls (Anhui and combined subjects)

\begin{tabular}{|c|c|c|c|c|c|c|c|c|c|c|}
\hline \multirow[t]{3}{*}{ Variables } & \multicolumn{5}{|c|}{ Anhui province } & \multicolumn{5}{|c|}{ Combined subjects } \\
\hline & \multicolumn{2}{|c|}{ Cases (N=119) } & \multicolumn{2}{|c|}{ Controls $(\mathrm{N}=264)$} & \multirow[t]{2}{*}{$P^{a}$} & \multicolumn{2}{|c|}{ Cases $(\mathrm{N}=512)$} & \multicolumn{2}{|c|}{ Controls (N=1076) } & \multirow[t]{2}{*}{$P^{a}$} \\
\hline & No. & $\%$ & No. & $\%$ & & No. & $\%$ & No. & $\%$ & \\
\hline Age range, month & \multicolumn{2}{|l|}{$0.001-108$} & \multicolumn{2}{|l|}{$0.001-96$} & 0.507 & \multicolumn{2}{|c|}{$0-132$} & \multicolumn{2}{|c|}{$0.001-156$} & 0.937 \\
\hline Mean \pm SD & \multicolumn{2}{|l|}{$26.86 \pm 23.19$} & \multicolumn{2}{|c|}{$29.67 \pm 27.52$} & & \multicolumn{2}{|c|}{$33.82 \pm 26.96$} & \multicolumn{2}{|c|}{$33.70 \pm 28.68$} & \\
\hline$\leq 18$ & 52 & 43.70 & 125 & 47.35 & & 178 & 34.77 & 430 & 39.96 & \\
\hline$>18$ & 67 & 56.30 & 139 & 52.65 & & 334 & 65.23 & 646 & 60.04 & \\
\hline \multicolumn{11}{|l|}{ Gender } \\
\hline Female & 50 & 42.02 & 112 & 42.42 & 0.941 & 218 & 42.58 & 454 & 42.02 & 0.885 \\
\hline Male & 69 & 57.98 & 152 & 57.58 & & 294 & 57.42 & 622 & 57.98 & \\
\hline \multicolumn{11}{|l|}{ Clinical stages } \\
\hline I & 45 & 37.82 & & & & 114 & 22.31 & & & \\
\hline II & 52 & 43.70 & & & & 145 & 28.38 & & & \\
\hline III & 14 & 11.76 & & & & 82 & 16.05 & & & \\
\hline IV & 8 & 6.72 & & & & 151 & 29.55 & & & \\
\hline $4 \mathrm{~s}$ & 0 & 0.00 & & & & 11 & 2.15 & & & \\
\hline NA & 0 & 0.00 & & & & 8 & 1.57 & & & \\
\hline \multicolumn{11}{|l|}{ Sites of origin } \\
\hline Adrenal gland & 43 & 36.13 & & & & 196 & 38.28 & & & \\
\hline Retroperitoneal region & 41 & 34.45 & & & & 128 & 25.00 & & & \\
\hline Mediastinum & 26 & 21.85 & & & & 135 & 26.37 & & & \\
\hline Other region & 9 & 7.56 & & & & 45 & 8.79 & & & \\
\hline NA & 0 & 0.00 & & & & 8 & 1.56 & & & \\
\hline
\end{tabular}


Table 2. Associations between hOGG1 polymorphisms and neuroblastoma susceptibility

\begin{tabular}{|c|c|c|c|c|c|c|c|}
\hline Genotype & $\begin{array}{l}\text { Cases } \\
(N=512)\end{array}$ & $\begin{array}{l}\text { Controls } \\
(N=1076)\end{array}$ & $P$ a & $\begin{array}{l}\text { Crude OR } \\
(95 \% \text { CI })\end{array}$ & $P$ & $\begin{array}{l}\text { Adjusted OR } \\
(95 \% \mathrm{CI})^{\mathrm{b}}\end{array}$ & $P_{b}^{b}$ \\
\hline \multicolumn{8}{|c|}{ rs1052133 G>C } \\
\hline GG & 197 (38.48) & $330(30.67)$ & & 1.00 & & 1.00 & \\
\hline GC & $217(42.38)$ & $567(52.70)$ & & $0.64(0.51-0.81)$ & 0.0002 & $0.64(0.51-0.81)$ & 0.0002 \\
\hline $\mathrm{CC}$ & $98(19.14)$ & $179(16.64)$ & & $0.92(0.68-1.24)$ & 0.576 & $0.92(0.68-1.24)$ & 0.583 \\
\hline Additive & & & 0.0005 & $0.90(0.77-1.04)$ & 0.155 & $0.90(0.77-1.04)$ & 0.157 \\
\hline Dominant & 315 (30.67) & $746(69.33)$ & 0.002 & $0.71(0.57-0.88)$ & 0.002 & $0.71(0.57-0.88)$ & 0.002 \\
\hline Recessive & $414(80.86)$ & $897(83.36)$ & 0.219 & $1.19(0.90-1.56)$ & 0.219 & $1.19(0.90-1.56)$ & 0.216 \\
\hline \multicolumn{8}{|c|}{ rs159153 $\mathrm{T}>\mathrm{C}$} \\
\hline TT & 415 (81.05) & 858 (79.74) & & 1.00 & & 1.00 & \\
\hline $\mathrm{TC}$ & $94(18.36)$ & $211(19.61)$ & & $0.92(0.70-1.21)$ & 0.550 & $0.92(0.70-1.21)$ & 0.553 \\
\hline $\mathrm{CC}$ & $3(0.59)$ & $7(0.65)$ & & $0.89(0.23-3.44)$ & 0.861 & $0.89(0.23-3.46)$ & 0.866 \\
\hline Additive & & & 0.827 & $0.92(0.72-1.19)$ & 0.540 & $0.92(0.72-1.19)$ & 0.544 \\
\hline Dominant & 97 (18.95) & $218(20.26)$ & 0.539 & $0.92(0.71-1.20)$ & 0.539 & $0.92(0.71-1.20)$ & 0.543 \\
\hline Recessive & 509 (99.41) & 1069 (99.35) & 0.879 & $0.90(0.23-3.50)$ & 0.881 & $0.91(0.23-3.52)$ & 0.886 \\
\hline \multicolumn{8}{|c|}{ rs293795 A>G } \\
\hline AA & $462(90.23)$ & 955 (88.75) & & 1.00 & & 1.00 & \\
\hline AG & $49(9.57)$ & $116(10.78)$ & & $0.87(0.61-1.24)$ & 0.450 & $0.87(0.61-1.24)$ & 0.451 \\
\hline GG & $1(0.20)$ & $5(0.46)$ & & $0.41(0.05-3.55)$ & 0.421 & $0.42(0.05-3.57)$ & 0.423 \\
\hline Additive & & & 0.538 & $0.85(0.61-1.18)$ & 0.319 & $0.85(0.61-1.18)$ & 0.321 \\
\hline Dominant & $50(9.77)$ & $121(11.25)$ & 0.374 & $0.85(0.60-1.21)$ & 0.374 & $0.86(0.60-1.21)$ & 0.376 \\
\hline Recessive & $511(99.80)$ & $1071(99.54)$ & 0.414 & $0.42(0.05-3.60)$ & 0.428 & $0.42(0.05-3.62)$ & 0.430 \\
\hline \multicolumn{8}{|c|}{ Combined effect of protective genotypes } \\
\hline 0 & $180(35.16)$ & $311(28.90)$ & & 1.00 & & 1.00 & \\
\hline 1 & $230(44.92)$ & $512(47.58)$ & & $0.78(0.61-0.99)$ & 0.039 & $0.78(0.61-0.99)$ & 0.039 \\
\hline 2 & $74(14.45)$ & 186 (17.29) & & $0.69(0.50-0.95)$ & 0.024 & $0.69(0.50-0.95)$ & 0.024 \\
\hline 3 & $28(5.47)$ & $67(6.23)$ & & $0.72(0.45-1.16)$ & 0.182 & $0.72(0.45-1.17)$ & 0.184 \\
\hline $1-3$ & 332 (64.84) & 765 (71.10) & 0.012 & $0.75(0.60-0.94)$ & 0.012 & $0.75(0.60-0.94)$ & 0.012 \\
\hline
\end{tabular}

$\mathrm{OR}$, odds ratio; $\mathrm{CI}$, confidence interval. a $\chi^{2}$ test for genotype distributions between neuroblastoma patients and controls. ${ }^{\mathrm{b}}$ Adjusted for age and gender. ${ }^{\mathrm{c}}$ Protective genotypes were rs1052133 GC/CC, rs159153 TC/CC, rs293795 AG/GG.

Table 3. Stratification analysis for association between hOGGI genotypes and neuroblastoma susceptibility

\begin{tabular}{|c|c|c|c|c|c|c|c|c|}
\hline \multirow[t]{2}{*}{ Variables } & \multicolumn{2}{|c|}{$\begin{array}{l}\text { rs1052133 } \\
\text { (cases/controls) }\end{array}$} & \multirow[t]{2}{*}{$\operatorname{AOR}(95 \% \mathrm{CI})^{a}$} & \multirow[t]{2}{*}{$P$ a } & \multicolumn{2}{|c|}{$\begin{array}{l}\text { Protective genotype } \\
\text { (cases/controls) }\end{array}$} & \multirow[t]{2}{*}{$\operatorname{AOR}(95 \% \mathrm{CI})^{\mathrm{a}}$} & \multirow[t]{2}{*}{$P$ a } \\
\hline & GG & GC/CC & & & 0 & $1-3$ & & \\
\hline \multicolumn{9}{|l|}{ Age, month } \\
\hline$\leq 18$ & $65 / 136$ & $113 / 294$ & $0.80(0.56-1.16)$ & 0.238 & $60 / 128$ & $118 / 302$ & $0.83(0.57-1.21)$ & 0.331 \\
\hline$>18$ & $132 / 194$ & $202 / 452$ & $0.66(0.50-0.87)$ & 0.003 & $120 / 183$ & $214 / 463$ & $0.71(0.53-0.93)$ & 0.015 \\
\hline \multicolumn{9}{|l|}{ Gender } \\
\hline Females & $81 / 149$ & $137 / 305$ & $0.83(0.59-1.16)$ & 0.279 & $77 / 139$ & $141 / 315$ & $0.81(0.58-1.14)$ & 0.230 \\
\hline Males & $116 / 181$ & $178 / 441$ & $0.63(0.47-0.85)$ & 0.002 & $103 / 172$ & $191 / 450$ & $0.71(0.53-0.96)$ & 0.026 \\
\hline \multicolumn{9}{|l|}{ Sites of origin } \\
\hline Adrenal gland & $68 / 330$ & $128 / 746$ & $0.84(0.61-1.15)$ & 0.275 & $66 / 311$ & $130 / 765$ & $0.81(0.58-1.12)$ & 0.192 \\
\hline Retroperitoneal & $55 / 330$ & $73 / 746$ & $0.58(0.40-0.85)$ & 0.005 & $50 / 311$ & $78 / 765$ & $0.63(0.43-0.92)$ & 0.017 \\
\hline Mediastinum & $54 / 330$ & $81 / 746$ & $0.66(0.46-0.96)$ & 0.029 & $46 / 311$ & $89 / 765$ & $0.79(0.54-1.15)$ & 0.213 \\
\hline Others & $15 / 330$ & $30 / 746$ & $0.88(0.47-1.67)$ & 0.703 & $13 / 311$ & $32 / 765$ & $1.00(0.52-1.93)$ & 0.991 \\
\hline \multicolumn{9}{|l|}{ Clinical stages } \\
\hline $\mathrm{I}+\mathrm{II}+4 \mathrm{~s}$ & $106 / 330$ & $153 / 746$ & $0.64(0.48-0.85)$ & 0.002 & $94 / 311$ & $165 / 765$ & $0.71(0.54-0.95)$ & 0.020 \\
\hline III+IV & $83 / 330$ & $150 / 746$ & $0.81(0.60-1.09)$ & 0.155 & $79 / 311$ & $154 / 765$ & $0.80(0.59-1.08)$ & 0.145 \\
\hline
\end{tabular}

AOR, Adjusted odds ratio; CI, confidence interval.

a Adjusted for age and gender, without the corresponding factor.

Herein, we for the first time investigated whether hOGG1 gene SNPs could affect the risk of neuroblastoma in Chinese children. The rs1052133 $\mathrm{G}>\mathrm{C}$, also referred as Ser326Cys, was located in exon 7. This genetic variant could result in an amino acid substitution of serine (Ser) with cysteine (Cys) at codon 326. It was reported by Kohno et al. that the substitution of amino acid affects hOGG1 function and further reduces DNA repair activity in an in vitro functional complementation assay [25]. Our results showed that the rs1052133 GC (Cys/Ser) was associated with decreased neuroblastoma risk, when comparing to GG (Cys/Cys) genotype. The protective role of rs1052133 C (Ser) allele was also detected in other cancer types. For example, Xing et al. found that rs1052133 CC homozygotes confer to decreased risk for developing esophageal squamous cell carcinoma in a Chinese population [39]. Chen et al. observed that subjects possessing the hOGG1 rs1052133 C allele were less likely to develop prostate cancer in comparison with those carrying the $G$ allele [40]. To note, our results failed to verify the hypothesis that 
the CC genotype is associated with decreased risk to neuroblastoma. This may be potentially attributed to relatively moderate sample size. Besides, the final effect of hOGG1 gene SNPs on neuroblastoma risk could be influenced by other exposure factors such as smoking status. Null relationship between SNP rs1052133 G>C and cancer risk was also observed. In a population-based (245 cases and 222 controls) and family-based (159 hereditary prostate cancer families) study, $\mathrm{Xu}$ et al. failed to detect the association between hOGG1 rs1052133 G>C and prostate cancer risk [41]. Wikman et al. did not find statistical differences in rs1052133 genotype distribution between lung cancer cases and normal controls [42].

The current study failed to establish a relationship between rs159153 $\mathrm{T}>\mathrm{C}$, rs293795 A>G polymorphisms and neuroblastoma risk. This could be explained by that some weak impact single polymorphism in each gene might not be strong enough to influence the risk of cancer. Individual analysis of rs1052133 G>C, rs159153 T>C, rs293795 $A>G$ revealed that only rs1052133 $G>C$ polymorphism may be associated with decreased risk of neuroblastoma significantly. However, rs159153 TC/CC, rs293795 AG/GG genotypes may be associated with decreased risk of neuroblastoma, but not in significant level. Thus, we considered rs1052133 GC/CC, rs159153 TC/CC, and rs293795 AG/GG as protective genotypes, and further analyzed their combined effect. We found that individuals carrying $1,2,1-3$ protective genotypes have a significant decreased neuroblastoma risk in comparison to those without protective genotypes.

In the stratified analysis, the significant association between rs1052133 genotype and neuroblastoma susceptibility was observed in neuroblastoma originating from retroperitoneal and mediastinum. However, the association of 1-3 protective genotypes with neuroblastoma susceptibility was detected only in neuroblastoma originating from retroperitoneal. Such discrepancy might be attributed to the small sample size in the stratified analysis.

Advantages of our study include the relatively large sample size as well as our analysis of three independent populations. Several limitations should be noted. First, although this is a multi-center study, the sample size is relatively moderate. Therefore, statistical power was impaired, especially for the stratification analysis. Second, only genetic factors were investigated; other environmental factors and genetic-environmental factors should also be included in the further study. Third, as this is a retrospective study, information bias and selection bias were inevitable. We have reduced these biases by frequency matching of cases and controls by age and gender. However, other important information such as parental exposures, living environment, and dietary intake was unavailable. Thus, this information should be included in the further prospective studies. Last, although the participants were enrolled from three regions, they were all Han Chinese. The conclusions should be interpreted with caution when extrapolated to other ethnic groups.

In conclusion, our results suggest that polymorphism rs1052133 G>C in hOGG1 gene was significantly associated with decreased neuroblastoma risk, in the Chinese population studied. Further functional studies are warranted to elucidate the biological role of $h O G G 1$ gene SNPs in the etiology of neuroblastoma.

\section{Abbreviations}

GWAS: genome-wide association study; 8OH-dG: 8-hydroxy-2-deoxyguanine; hOGG1: human 8-oxoguanine glycosylase 1; SNP: single nucleotide polymorphism; OR: odds ratio; CI: confidence interval; Ser: serine; Cys: cysteine.

\section{Acknowledgments}

This work was funded by grants from the Pearl River S\&T Nova Program of Guangzhou (No: 201710010086), and the State Clinical Key Specialty Construction Project (Pediatric Surgery) 2013 (No: GJLCZD1301).

\section{Competing Interests}

The authors have declared that no competing interest exists.

\section{References}

1. Capasso M, Diskin SJ. Genetics and genomics of neuroblastoma. Cancer Treat Res. 2010; 155: 65-84.

2. Matthay KK, Maris JM, Schleiermacher G, Nakagawara A, Mackall CL, Diller L, et al. Neuroblastoma. Nat Rev Dis Primers. 2016; 2: 16078.

3. Maris JM, Hogarty MD, Bagatell R, Cohn SL. Neuroblastoma. Lancet. 2007; 369: 2106-20.

4. Maris JM. Recent advances in neuroblastoma. N Engl J Med. 2010; 362: 2202-11.

5. Esposito MR, Aveic S, Seydel A, Tonini GP. Neuroblastoma treatment in the post-genomic era. J Biomed Sci. 2017; 24: 14.

6. Cheung NK, Dyer MA. Neuroblastoma: developmental biology, cancer genomics and immunotherapy. Nat Rev Cancer. 2013; 13: 397-411.

7. Irwin MS, Park JR. Neuroblastoma: paradigm for precision medicine. Pediatr Clin North Am. 2015; 62: 225-56.

8. Menegaux F, Olshan AF, Neglia JP, Pollock BH, Bondy ML. Day care, childhood infections, and risk of neuroblastoma. Am J Epidemiol. 2004; 159: 843-51.

9. Cook MN, Olshan AF, Guess HA, Savitz DA, Poole C, Blatt J, et al. Maternal medication use and neuroblastoma in offspring. Am J Epidemiol. 2004; 159: 721-31.

10. Mosse YP, Laudenslager M, Khazi D, Carlisle AJ, Winter CL, Rappaport E, et al. Germline PHOX2B mutation in hereditary neuroblastoma. Am J Hum Genet. 2004; 75: 727-30

11. Chen Y, Takita J, Choi YL, Kato M, Ohira M, Sanada M, et al. Oncogenic mutations of ALK kinase in neuroblastoma. Nature. 2008; 455: 971-4.

12. Mosse $\mathrm{YP}$, Laudenslager M, Longo L, Cole KA, Wood A, Attiyeh EF, et al. Identification of ALK as a major familial neuroblastoma predisposition gene. Nature. 2008; 455: 930-5. 
13. Diskin SI, Capasso M, Diamond M, Oldridge DA, Conkrite K, Bosse KR, et al. Rare variants in TP53 and susceptibility to neuroblastoma. J Natl Cancer Inst. 2014; 106: dju047.

14. Diskin SJ, Capasso M, Schnepp RW, Cole KA, Attiyeh EF, Hou C, et al. Common variation at $6 \mathrm{q} 16$ within HACE1 and LIN28B influences susceptibility to neuroblastoma. Nat Genet. 2012; 44: 1126-30.

15. Maris JM, Mosse YP, Bradfield JP, Hou C, Monni S, Scott RH, et al. Chromosome $6 \mathrm{p} 22$ locus associated with clinically aggressive neuroblastoma. N Engl J Med. 2008; 358: 2585-93.

16. Capasso M, Devoto M, Hou C, Asgharzadeh S, Glessner JT, Attiyeh EF, et al. Common variations in BARD1 influence susceptibility to high-risk neuroblastoma. Nat Genet. 2009; 41: 718-23.

17. He J, Zou Y, Wang T, Zhang R, Yang T, Zhu J, et al. Genetic Variations of GWAS-Identified Genes and Neuroblastoma Susceptibility: a Replication Study in Southern Chinese Children. Transl Oncol. 2017; 10: 936-41.

18. Basu AK. DNA Damage, Mutagenesis and Cancer. Int J Mol Sci. 2018; 19.

19. Asami S, Manabe H, Miyake J, Tsurudome $Y$, Hirano T, Yamaguchi R, et al. Cigarette smoking induces an increase in oxidative DNA damage, 8-hydroxydeoxyguanosine, in a central site of the human lung. Carcinogenesis. 1997; 18: 1763-6.

20. Helbock HJ, Beckman KB, Shigenaga MK, Walter PB, Woodall AA, Yeo HC, et al. DNA oxidation matters: the HPLC-electrochemical detection assay of 8-oxo-deoxyguanosine and 8-oxo-guanine. Proc Natl Acad Sci U S A. 1998; 95 : 288-93.

21. Shibutani S, Takeshita M, Grollman AP. Insertion of specific bases during DNA synthesis past the oxidation-damaged base 8-oxodG. Nature. 1991; 349: 431-4

22. Kasai $H$. Analysis of a form of oxidative DNA damage, 8-hydroxy-2'-deoxyguanosine, as a marker of cellular oxidative stress during carcinogenesis. Mutat Res. 1997; 387: 147-63.

23. Shinmura K, Yokota J. The OGG1 gene encodes a repair enzyme for oxidatively damaged DNA and is involved in human carcinogenesis. Antioxid Redox Signal. 2001; 3: 597-609.

24. Roldan-Arjona T, Wei YF, Carter KC, Klungland A, Anselmino C, Wang RP, et al. Molecular cloning and functional expression of a human cDNA encoding the antimutator enzyme 8-hydroxyguanine-DNA glycosylase. Proc Natl Acad Sci U S A. 1997; 94: 8016-20.

25. Kohno T, Shinmura K, Tosaka M, Tani M, Kim SR, Sugimura H, et al. Genetic polymorphisms and alternative splicing of the hOGG1 gene, that is involved in the repair of 8-hydroxyguanine in damaged DNA. Oncogene. 1998; 16: 3219-25.

26. Ban EZ, Lye MS, Chong PP, Yap YY, Lim SYC, Abdul Rahman H. Haplotype CGC from XPD, hOGG1 and ITGA2 polymorphisms increases the risk of nasopharyngeal carcinoma in Malaysia. PLoS One. 2017; 12: e0187200.

27. Weiss JM, Goode EL, Ladiges WC, Ulrich CM. Polymorphic variation in hOGG1 and risk of cancer: a review of the functional and epidemiologic literature. Mol Carcinog. 2005; 42: 127-41.

28. Zhang J, Zhuo ZJ, Wang J, He J, Yang L, Zhang D, et al. CASC15 gene polymorphisms reduce neuroblastoma risk in Chinese children. Oncotarget. 2017; 8: 91343-9.

29. He J, Zhang X, Zhang J, Zhang R, Yang T, Zhu J, et al. LMO1 super-enhancer polymorphism rs2168101 G>T correlates with decreased neuroblastoma risk in Chinese children. J Cancer. 2018; 9: 1592-7.

30. Wu Q, Zhuo ZJ, Zeng J, Zhang J, Zhu J, Zou Y, et al. Association between NEFL Gene Polymorphisms and Neuroblastoma Risk in Chinese Children: A Two-Center Case-Control Study. J Cancer. 2018; 9: 535-9.

31. He J, Wang F, Zhu J, Zhang Z, Zou Y, Zhang R, et al. The TP53 gene rs1042522 $\mathrm{C}>\mathrm{G}$ polymorphism and neuroblastoma risk in Chinese children. Aging (Albany NY). 2017; 9: 852-9.

32. Zhang J, Lin H, Wang J, He J, Zhang D, Qin P, et al. LMO1 polymorphisms reduce neuroblastoma risk in Chinese children: a two-center case-control study. Oncotarget. 2017; 8: 65620-6.

33. He J, Zou Y, Liu X, Zhu J, Zhang J, Zhang R, et al. Association of Common Genetic Variants in Pre-microRNAs and Neuroblastoma Susceptibility: A Two-Center Study in Chinese Children. Mol Ther Nucleic Acids. 2018; 11:1-8.

34. He J, Qiu LX, Wang MY, Hua RX, Zhang RX, Yu HP, et al. Polymorphisms in the XPG gene and risk of gastric cancer in Chinese populations. Hum Genet. 2012; 131: 1235-44

35. He J, Wang F, Zhu J, Zhang R, Yang T, Zou Y, et al. Association of potentially functional variants in the XPG gene with neuroblastoma risk in a Chinese population. J Cell Mol Med. 2016; 20: 1481-90.

36. He J, Zhuo ZJ, Zhang A, Zhu J, Hua RX, Xue WQ, et al. Genetic variants in the nucleotide excision repair pathway genes and gastric cancer susceptibility in a southern Chinese population. Cancer Manag Res. 2018; 10: 765-74.

37. Zhuo ZJ, Zhang R, Zhang J, Zhu J, Yang T, Zou Y, et al. Associations between lncRNA MEG3 polymorphisms and neuroblastoma risk in Chinese children. Aging (Albany NY). 2018; 10: 481-91.

38. Zhuo ZJ, Liu W, Zhang J, Zhu J, Zhang R, Tang J, et al. Functional Polymorphisms at ERCC1/XPF Genes Confer Neuroblastoma Risk in Chinese Children. EBioMedicine. 2018; 30: 113-9.

39. Xing DY, Tan W, Song N, Lin DX. Ser326Cys polymorphism in hOGG1 gene and risk of esophageal cancer in a Chinese population. Int J Cancer. 2001:95: $140-3$
40. Chen L, Elahi A, Pow-Sang J, Lazarus P, Park J. Association between polymorphism of human oxoguanine glycosylase 1 and risk of prostate cancer. J Urol. 2003; 170: 2471-4.

41. Xu J, Zheng SL, Turner A, Isaacs SD, Wiley KE, Hawkins GA, et al. Associations between hOGG1 sequence variants and prostate cancer susceptibility. Cancer Res. 2002; 62: 2253-7.

42. Wikman H, Risch A, Klimek F, Schmezer P, Spiegelhalder B, Dienemann H, et al. hOGG1 polymorphism and loss of heterozygosity (LOH): significance for lung cancer susceptibility in a caucasian population. Int J Cancer. 2000; 88: $932-7$ 